\title{
Accurate Time-Domain Modeling of Reconfigurable Antenna Sensors for Non-Invasive Melanoma Skin Cancer Detection
}

\author{
Diego Caratelli, Alessandro Massaro, Roberto Cingolani, and Alexander G. Yarovoy, Senior Member, IEEE
}

\begin{abstract}
The full-wave electromagnetic characterization of reconfigurable antenna sensors for non-invasive detection of melanoma-related anomalies of the skin is presented. To this end, an enhanced locally conformal finite-difference time-domain procedure, based on the definition of effective material parameters and a suitable normalization of the electromagnetic field-related quantities, is adopted. In this way, an insightful understanding of the physical processes responsible for the performance of considered class of devices is achieved. This in turn is important in order to enhance the structure reliability, optimizing the design cycle. A suitable microelectromechanical-system-based sensor layout is finally discussed in details.
\end{abstract}

Index Terms-Locally conformal finite-difference time-domain (FDTD) modeling, non-invasive melanoma skin cancer detection, reconfigurable antenna sensors, wireless telemetry.

\section{INTRODUCTION}

$\mathbf{R}$ ECENTLY, microelectromechanical-system (MEMS) antennas have drawn significant interest in the design of high-performance communication devices [1]-[7], and sensors for wireless telemetry applications [8]-[10]. In particular, microsensors can be conveniently implanted in the interstitial fluid under the skin in order to monitor physiological parameters such as temperature, blood pressure, etc. As it can be readily inferred, antennas play the major role in implantable sensing devices since they directly affect the radio link with the external equipment.

The design of implantable telemetry systems is very challenging due to demanding requirements in terms of reduced volume occupation, good impedance matching property, low power consumption, and biocompatibility with the human body. Furthermore, the host environment consisting of biological tissues adds significant complexity to the problem due to the high losses.

Manuscript received November 15, 2010; revised January 12, 2011; accepted February 12, 2011. Date of publication February 22, 2011; date of current version February 03, 2012. This work was partly carried out in the framework of the STARS project funded by the Dutch government. The associate editor coordinating the review of this paper and approving it for publication was Prof. Aime Lay-Ekuakille.

D. Caratelli and A. G. Yarovoy are with the Delft University of Technology, IRCTR, 2628 CD Delft, The Netherlands (e-mail: d.caratelli@tudelft.nl; o.yarovyi@ tudelft.nl).

A. Massaro and R. Cingolani are with the Center of Bio-Molecular Nanotechnology, Italian Institute of Technology, Arnesano 73100, Italy (e-mail: alessandro.massaro@iit.it; roberto.cingolani@iit.it).

Color versions of one or more of the figures in this paper are available online at http://ieeexplore.ieee.org.

Digital Object Identifier 10.1109/JSEN.2011.2117417
In this paper, attention is put on the design of biocompatible microprobes for the detection of melanoma-related anomalies of the skin. Melanoma is a serious form of skin cancer originating in the pigment-producing cells (melanocytes). These cells become abnormal, grow uncontrollably, and aggressively invade surrounding tissues. The skin has several layers, but the main layers are the dermis (lower or inner layer), and the epidermis (upper or outer layer) where skin cancers originate, and which is made up of three types of cells.

- Squamous cells: Thin, flat cells that form the top layer of the epidermis.

- Basal cells: Round cells under the squamous cells.

- Melanocytes: Found in the lower part of the epidermis, these cells produce melanin, the pigment that gives skin its natural color.

The growth of the melanoma results in a local variation of permittivity and electrical conductivity of the skin tissue, which can be monitored to estimate the extension of anomalous cells as function of time. In particular, the evolution of the melanoma can be analyzed by using the non-invasive microsensor system sketched in Fig. 1, where a modulated Gaussian pulse impinging on the skin surface interacts with the target. This interaction results in a diffracted electromagnetic field which is measured at the input terminals of the receive antenna. By changing the location of the probe and recording the device response as function of time (or frequency) and the sensor location, one collects the scattering data which can be processed to get a radargram of the target. The design and full-wave characterization of the considered class of devices is carried out by means of a dedicated locally conformal finite-difference time-domain (FDTD) approach for accurate modeling of complex electromagnetic structures. In this way, a detailed and insightful understanding of the physical processes responsible for the performance of reconfigurable antenna sensors is achieved. This in turn is very important in order to enhance the structure reliability, optimizing the design cycle. A suitable MEMS-based layout for the proposed microsensors is finally discussed in detail.

\section{TReatment And Detection TechniQues OF SKIN CANCER}

In previous works, the detection and treatment of the skin cancer have been conveniently addressed by means of suitable implantable microwave probes [11]. In fact, a very promising approach in the treatment of melanoma consists in the combined effect of heat and electromagnetic field radiation resulting 


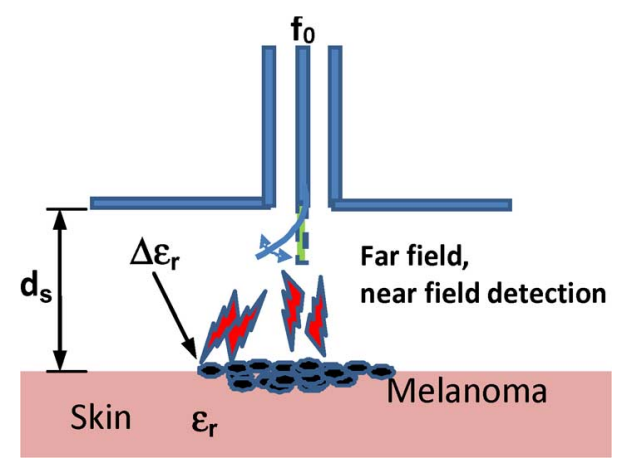

Fig. 1. Detection of a superficial skin cancer by means of a reconfigurable MEMS-based antenna sensor.

in a localized thermal effect within the cancerous tissue to be removed. On the other hand, a different layout, similar to the one sketched in Fig. 1, has been considered in [12] where a diagnostic procedure for the detection of the skin cancer based on microwave reflectometry is presented. Within such a procedure open-ended coaxial probes are adopted to measure the electrical properties of the skin. In this way, the detection of malignant lesions can be performed by evaluating variations on the average magnitude of the reflection coefficient at the input terminals of the probe. Recently, implantable sensors consisting of minimally invasive micro needle geometries [13], and optical-fiber probes for the measurement of the absorption spectrum of the human skin [14] have been also proposed. Furthermore, different signal processing approaches for the detection of melanoma are currently available in the scientific literature [15], [16]. One of these is based on Terahertz spectroscopy [15], which allows evaluating the local refractive index of the skin and, hence, the anomalies due to a possible melanoma. Another approach is based on the use of suitable implanted resonating antenna sensors [16]. In fact, the variation of the relevant resonant frequency due to the presence of a bio-target provides an effective means for the detection of a possible skin cancer. In this paper, a combined approach is proposed in order to achieve an enhanced detectability of melanomas. In particular, reconfigurable MEMS-based monopole-like antenna sensors operating at Terahertz frequencies are used to measure the variations of permittivity caused by the presence of anomalous cells on the skin surface.

\section{LOCALLY CONFORMAL FDTD TECHNIQUE}

The analysis and design of complex radiating structures requires accurate electromagnetic field prediction models. One such widely used technique is the FDTD algorithm. However, in the conventional formulation proposed by Yee [17], [18], each cell of the computational grid is implicitly supposed to be filled by a homogeneous material. For this reason, the adoption of Cartesian meshes could result in reduced numerical accuracy when structures having curved boundaries have to be modeled. In this case, locally conformal FDTD schemes [19] provide clear advantages over the use of the stair-casing approach or unstructured and stretched space lattices, potentially suffering from significant numerical dispersion and/or instability [18]. Such schemes, necessary to improve the numerical accuracy of the conventional algorithm, are based on the definition of effec-

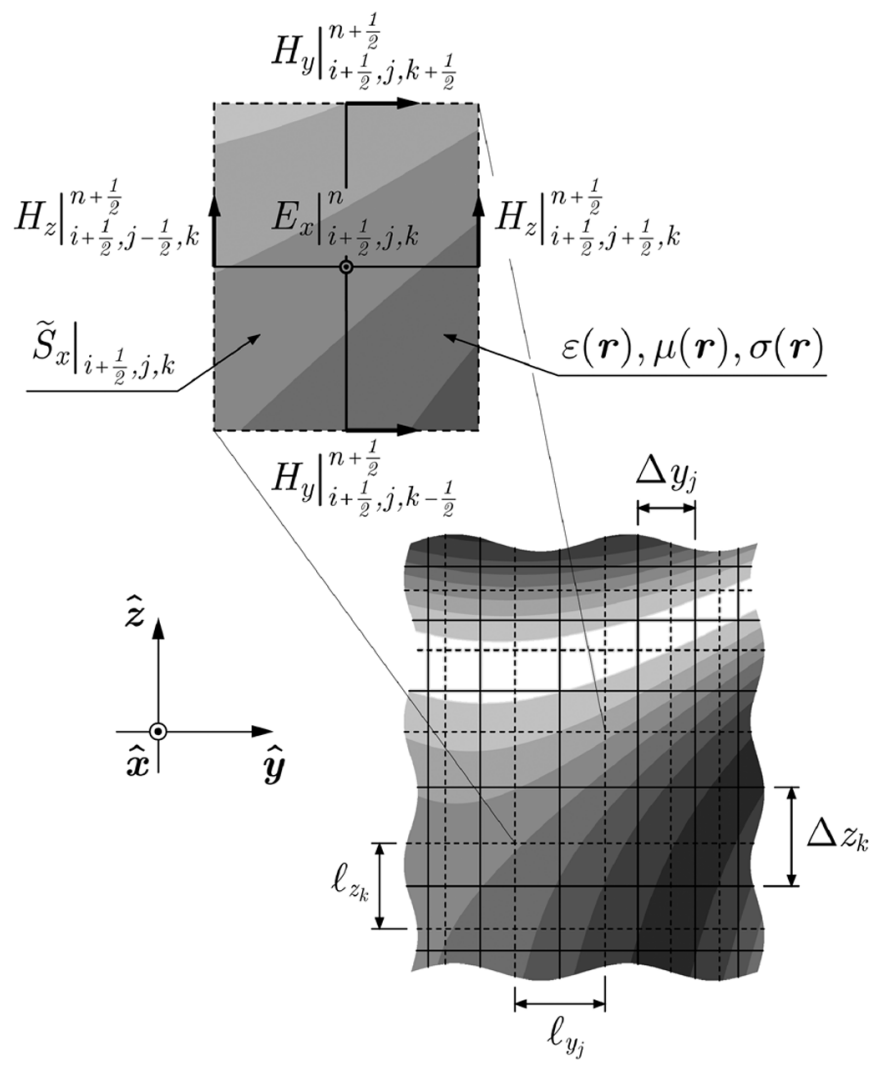

Fig. 2. Cross-sectional view of the FDTD computational grid in presence of curved boundaries between different dielectric materials.

tive material parameters suitable to describe the geometrical and electrical characteristics of the structure under analysis.

In this section, a computationally enhanced formulation of the locally conformal FDTD scheme proposed in [19] is described. To this end, let us consider a three-dimensional domain $D$ filled by a linear, isotropic, non-dispersive material, having permittivity $\varepsilon(\boldsymbol{r})$, magnetic permeability $\mu(\boldsymbol{r})$, and electrical conductivity $\sigma(\boldsymbol{r})$. In such a domain, a dual-space, non-uniform lattice formed by a primary and secondary mesh is introduced. The primary mesh $M_{D}$ is composed of space-filling hexahedrons, whose vertices are defined by the Cartesian coordinates

$$
\left\{\left(x_{i}, y_{j}, z_{k}\right) \mid i=0, \ldots, N_{x} ; j=0, \ldots, N_{y} ; k=0, \ldots, N_{z}\right\} .
$$

As a consequence, the edge lengths between adjacent vertices in $M_{D}$ result to be expressed as

$$
\begin{cases}\Delta x_{i}=x_{i+1}-x_{i}, & i=0,1, \ldots, N_{x}-1 \\ \Delta y_{j}=y_{j+1}-y_{j}, & j=0,1, \ldots, N_{y}-1 \\ \Delta z_{k}=z_{k+1}-z_{k}, & k=0,1, \ldots, N_{z}-1 .\end{cases}
$$

The secondary or dual mesh $\tilde{M}_{D}$ (see Fig. 2) is composed of the closed hexahedrons whose edges penetrate the shared faces of the primary cells and connect the relevant centroids, having coordinates $x_{i+1 / 2}=x_{i}+\Delta x_{i} / 2, y_{j+1 / 2}=y_{j}+\Delta y_{j} / 2$, $z_{k+1 / 2}=z_{k}+\Delta z_{k} / 2$. A set of dual edge lengths is then introduced in $\tilde{M}_{D}$ as follows:

$$
\begin{cases}\ell_{x_{i}}=\left(\Delta x_{i-1}+\Delta x_{i}\right) / 2, & i=1,2, \ldots, N_{x}-1 \\ \ell_{y_{j}}=\left(\Delta y_{j-1}+\Delta y_{j}\right) / 2, & j=1,2, \ldots, N_{y}-1 \\ \ell_{z_{k}}=\left(\Delta z_{k-1}+\Delta z_{k}\right) / 2, & k=1,2, \ldots, N_{z}-1 .\end{cases}
$$


As usual, the electric field components are defined along each edge of a primary lattice cell, whereas the magnetic field components are assumed to be located along the edges of the secondary lattice cells. In this formulation, the relationship between $E$ - and $H$-field components is given by Maxwell's equations expressed in integral form, specifically using Faraday-Neumann's law and Ampere's law, respectively. In particular, the enforcement of the Ampere's law on the generic dual-mesh cell surface $\left.\tilde{S}_{x}\right|_{i+1 / 2, j, k}$ having boundary $\left.\partial \tilde{S}_{x}\right|_{i+1 / 2, j, k}=\left.\tilde{C}_{x}\right|_{i+1 / 2, j, k}$ (see Fig. 2) results in the following integral equation:

$$
\begin{aligned}
\oint_{\left.\tilde{C}_{x}\right|_{i+1 / 2, j, k}} \boldsymbol{H}(\boldsymbol{r}, t) \cdot \boldsymbol{d l}= & \iint_{\left.\tilde{S}_{x}\right|_{i+1 / 2, j, k}} \sigma(\boldsymbol{r}) E_{x}(\boldsymbol{r}, t) d S \\
& +\frac{\partial}{\partial t} \underset{\left.\tilde{S}_{x}\right|_{i+1 / 2, j, k}}{\iint_{i}} \varepsilon(\boldsymbol{r}) E_{x}(\boldsymbol{r}, t) d S
\end{aligned}
$$

where

$$
\begin{gathered}
\oint_{\left.\tilde{C}_{x}\right|_{i+1 / 2, j, k}} \boldsymbol{H}(\boldsymbol{r}, t) \cdot \boldsymbol{d l} \\
=\ell_{z_{k}}\left[H_{z}\left(x_{i+\frac{1}{2}}, y_{j+\frac{1}{2}}, z_{k}, t\right)+\right. \\
\left.\quad-H_{z}\left(x_{i+\frac{1}{2}}, y_{j-\frac{1}{2}}, z_{k}, t\right)\right] \\
-\ell_{y_{j}}\left[H_{y}\left(x_{i+\frac{1}{2}}, y_{j}, z_{k+\frac{1}{2}}, t\right)+\right. \\
\left.\quad-H_{y}\left(x_{i+\frac{1}{2}}, y_{j}, z_{k-\frac{1}{2}}, t\right)\right] \\
+o\left(\ell_{y_{j}}\right)+o\left(\ell_{z_{k}}\right)
\end{gathered}
$$

as $\ell_{y_{j}}$ and $\ell_{z_{k}}$ tend to zero. Under the assumption that the spatial increments $\Delta x_{i}, \Delta y_{j}, \Delta z_{k}$ of the computational grid are small compared to the minimum working wavelength, the infinitesimal terms of higher order appearing in (5) can be neglected. Moreover, it should be noticed that the $x$-component of the electric field is continuous along the interfaces crossing $\left.\tilde{S}_{x}\right|_{i+1 / 2, j, k}$ so that, under the mentioned hypothesis, the following approximation can be made:

$$
\begin{aligned}
\iint_{\left.\tilde{S}_{x}\right|_{i+1 / 2, j, k}}\left\{\begin{array}{l}
\varepsilon \\
\sigma
\end{array}\right\}(\boldsymbol{r}) E_{x}(\boldsymbol{r}, t) d S \\
\simeq E_{x}\left(x_{i+\frac{1}{2}}, y_{j}, z_{k}, t\right) \underset{\left.\tilde{S}_{x}\right|_{i+1 / 2, j, k}}{\iint_{\sigma}}\left\{\begin{array}{l}
\varepsilon \\
\sigma
\end{array}\right\}(\boldsymbol{r}) d S .
\end{aligned}
$$

Hence, combining the equations above yields

$$
\begin{aligned}
\bar{\varepsilon}_{x}^{e f f} & \left.\left.\right|_{i+\frac{1}{2}, j, k} \frac{\partial}{\partial t} \mathcal{E}_{x}(t)\right|_{i+\frac{1}{2}, j, k}+\left.\left.\bar{\sigma}_{x}^{e f f}\right|_{i+\frac{1}{2}, j, k} \mathcal{E}_{x}(t)\right|_{i+\frac{1}{2}, j, k} \\
= & \left.\mathcal{H}_{z}(t)\right|_{i+\frac{1}{2}, j+\frac{1}{2}, k}-\left.\mathcal{H}_{z}(t)\right|_{i+\frac{1}{2}, j-\frac{1}{2}, k} \\
& -\left.\mathcal{H}_{y}(t)\right|_{i+\frac{1}{2}, j, k+\frac{1}{2}}+\left.\mathcal{H}_{y}(t)\right|_{i+\frac{1}{2}, j, k-\frac{1}{2}}
\end{aligned}
$$

where we have introduced the normalized field quantities

$$
\begin{aligned}
\left.\mathcal{E}_{x}(t)\right|_{i+\frac{1}{2}, j, k} & =\Delta x_{i} E_{x}\left(x_{i+\frac{1}{2}}, y_{j}, z_{k}, t\right) \\
\left.\mathcal{H}_{z}(t)\right|_{i+\frac{1}{2}, j+\frac{1}{2}, k} & =\ell_{z_{k}} H_{z}\left(x_{i+\frac{1}{2}}, y_{j+\frac{1}{2}}, z_{k}, t\right)
\end{aligned}
$$

$$
\left.\mathcal{H}_{y}(t)\right|_{i+\frac{1}{2}, j, k+\frac{1}{2}}=\ell_{y_{j}} H_{y}\left(x_{i+\frac{1}{2}}, y_{j}, z_{k-\frac{1}{2}}, t\right)
$$

and the averaged effective permittivity $\left.\bar{\varepsilon}_{x}^{e f f}\right|_{i+1 / 2, j, k}$, and conductivity $\left.\bar{\sigma}_{x}^{e f f}\right|_{i+1 / 2, j, k}$, defined as follows:

$$
\left.\left\{\begin{array}{l}
\bar{\varepsilon} \\
\bar{\sigma}
\end{array}\right\}_{x}^{e f f}\right|_{i+\frac{1}{2}, j, k}=\frac{1}{\Delta x_{i}} \int_{z_{k-1 / 2}}^{z_{k+1 / 2}} \int_{y_{j-1 / 2}}^{y_{j+1 / 2}}\left\{\begin{array}{l}
\varepsilon \\
\sigma
\end{array}\right\}\left(x_{i+\frac{1}{2}}, y, z\right) d y d z .
$$

The time derivative in (7) is then evaluated using a central-difference approximation that is second order-accurate if $E$ - and $H$-field components are staggered in time domain [18]. This results in the following explicit time-stepping relation:

$$
\begin{aligned}
\left.\mathcal{E}_{x}\right|_{i+\frac{1}{2}, j, k} ^{n+1}=\left.\left.\bar{\alpha}_{x}^{(E)}\right|_{i+\frac{1}{2}, j, k} \mathcal{E}_{x}\right|_{i+\frac{1}{2}, j, k} ^{n-1} & +\left.\left.\bar{\beta}_{x}^{(E)}\right|_{i+\frac{1}{2}, j, k}(\nabla \times \mathcal{H})_{x}\right|_{i+\frac{1}{2}, j, k} ^{n+\frac{1}{2}}
\end{aligned}
$$

where

$$
\begin{aligned}
\left.(\nabla \times \mathcal{H})_{x}\right|_{i+\frac{1}{2}, j, k} ^{n+\frac{1}{2}}=\left.\mathcal{H}_{z}\right|_{i+\frac{1}{2}, j+\frac{1}{2}, k} ^{n+\frac{1}{2}}-\left.\mathcal{H}_{z}\right|_{i+\frac{1}{2}, j-\frac{1}{2}, k} ^{n+\frac{1}{2}}+ \\
-\left.\mathcal{H}_{y}\right|_{i+\frac{1}{2}, j, k+\frac{1}{2}} ^{n+\frac{1}{2}}+\left.\mathcal{H}_{y}\right|_{i+\frac{1}{2}, j, k-\frac{1}{2}} ^{n+\frac{1}{2}}
\end{aligned}
$$

denotes the finite-difference expression of the normalized $x$-component of the magnetic field curl at the time step $n+1 / 2$. In (12), the information regarding the local physical and geometrical properties of the electromagnetic structure under analysis is transferred to the position-dependent coefficients

$$
\begin{aligned}
\left.\bar{\alpha}_{x}^{(E)}\right|_{i+\frac{1}{2}, j, k} & =\frac{1-\left.\bar{Q}_{x}^{\text {eff }}\right|_{i+\frac{1}{2}, j, k}}{1+\left.\bar{Q}_{x}^{e f f}\right|_{i+\frac{1}{2}, j, k}} \\
\left.\bar{\beta}_{x}^{(E)}\right|_{i+\frac{1}{2}, j, k} & =\frac{\Delta t /\left.\bar{\varepsilon}_{x}^{e f f}\right|_{i+\frac{1}{2}, j, k}}{1+\left.\bar{Q}_{x}^{e f f}\right|_{i+\frac{1}{2}, j, k}}
\end{aligned}
$$

with:

$$
\left.\bar{Q}_{x}^{e f f}\right|_{i+\frac{1}{2}, j, k}=\frac{\left.\bar{\sigma}_{x}^{e f f}\right|_{i+\frac{1}{2}, j, k} \Delta t}{\left.2 \bar{\varepsilon}_{x}^{e f f}\right|_{i+\frac{1}{2}, j, k}} .
$$

The update equations of the remaining components of the electric and magnetic field can be easily derived by permuting the spatial indices $i, j, k$ and applying the duality principle in the discrete space.

As it can be readily noticed, the computation of positiondependent coefficients (14)-(16) can be carried out before the FDTD-method time marching starts. As a consequence, unlike in conformal techniques based on stretched space lattices, no additional correction is required in the core of the numerical algorithm. Furthermore, the resulting FDTD update equations (12), (13) have a very convenient structure, leading to a $14 \%$ reduction of the number of floating-point operations needed to determine the unknown field quantities in the generic mesh cell compared to the Yee algorithm [17], [18]. It is also to be pointed out that the proposed scheme has the same numerical stability properties as the conventional FDTD formulation, although it 
introduces a significant improvement in accuracy over the staircasing approximation as well as alternative weighted averaging FDTD approaches [20]. In order to assess the effectiveness of the developed technique, several test cases have been considered. For the sake of brevity, only the computation of the fundamental resonant frequency of a metallic cavity loaded with a cylindrical dielectric resonator is presented in the Appendix. The obtained results clearly demonstrate the suitability of the proposed scheme to efficiently handle the problem of modeling antenna sensors for skin cancer detection, where the accurate characterization of complex metal-dielectric objects having irregular geometry (think about the shape of a typical melanoma) is required.

\section{Full-WaVe Analysis of Microantenna SENSORS}

The locally conformal FDTD approach detailed in Section III has been adopted to perform the full-wave analysis of the monopole-like antenna sensors useful to detect variations of the permittivity due to anomalous melanoma cells located on the skin surface (see Fig. 1). In this way, one can also retrieve information about the growth rate of a possible melanoma, which is a very important aspect regarding the monitoring of the risk. In the following numerical investigations the considered radiating structures are assumed to be at a distance $d_{s}=1.5 \mathrm{~mm}$ from the surface of the skin, modeled as a dielectric material having relative permittivity $\varepsilon_{r}=35.1$ and electrical conductivity $\sigma=3.72 \mathrm{~S} / \mathrm{m}$ (see Fig. 1). In particular, the electromagnetic field computations have been carried out on a non-uniform grid with maximum spatial increment $\Delta h_{\max }=\lambda_{c} / 25 \simeq 40 \mu \mathrm{m}$, where $\lambda_{c}$ is the free-space wavelength at the upper $-10 \mathrm{~dB}$ cutoff frequency $f_{c}=300 \mathrm{GHz}$ of the excitation voltage signal, which is the Gaussian voltage pulse described by the equation

$$
v_{g}(t)=V_{g} \exp \left[-\left(\frac{t-t_{0}}{t_{g}}\right)^{2}\right] u(t)
$$

where $V_{g}=1 \mathrm{mV}, t_{0}=10 t_{g}$, and

$$
t_{g}=\sqrt{\ln 10} /\left(\pi f_{c}\right) \simeq 1.61 \mathrm{ps}
$$

In (17) $u(t)$ denotes the usual Heaviside unit-step function. The source pulse is then coupled into the finite-difference equations used to update the time-domain electric field distribution within the antenna driving point as follows:

$$
\begin{aligned}
& \left.\mathcal{E}_{z}\right|_{i_{g}, j_{g}, k_{g}+\frac{1}{2}} ^{n+1}=\left.\left.\bar{\alpha}_{z}^{(E, g)}\right|_{i_{g}, j_{g}, k_{g}+\frac{1}{2}} \mathcal{E}_{z}\right|_{i_{g}, j_{g}, k_{g}+\frac{1}{2}} ^{n-1} \\
& \quad+\left.\bar{\beta}_{z}^{(E, g)}\right|_{i_{g}, j_{g}, k_{g}+\frac{1}{2}}\left[\left.(\nabla \times \mathcal{H})_{z}\right|_{i_{g}, j_{g}, k_{g}+\frac{1}{2}} ^{n+\frac{1}{2}}+\left.\mathcal{I}_{g}\right|^{n+\frac{1}{2}}\right]
\end{aligned}
$$

where

$$
\begin{gathered}
\left.\bar{\alpha}_{z}^{(E, g)}\right|_{i_{g}, j_{g}, k_{g}+\frac{1}{2}}=\frac{1-\left.\bar{Q}_{z}^{(E, g)}\right|_{i_{g}, j_{g}, k_{g}+\frac{1}{2}}}{1+\left.\bar{Q}_{z}^{(E, g)}\right|_{i_{g}, j_{g}, k_{g}+\frac{1}{2}}} \\
\left.\bar{\beta}_{z}^{(E, g)}\right|_{i_{g}, j_{g}, k_{g}+\frac{1}{2}}=\frac{\Delta t /\left.\bar{\varepsilon}_{z}^{e f f}\right|_{i_{g}, j_{g}, k_{g}+\frac{1}{2}}}{1+\left.\bar{Q}_{z}^{(E, g)}\right|_{i_{g}, j_{g}, k_{g}+\frac{1}{2}}}
\end{gathered}
$$

with

$$
\left.\bar{Q}_{z}^{(E, g)}\right|_{i_{g}, j_{g}, k_{g}+\frac{1}{2}}=\frac{\Delta t}{\left.2 \bar{\varepsilon}_{z}^{e f f}\right|_{i_{g}, j_{g}, k_{g}+\frac{1}{2}} R_{g}}
$$

$R_{g}$ denoting the internal resistance of the voltage generator, and $i_{g}, j_{g}, k_{g}$ the spatial indices relevant to the feed point. In (19), the term

$$
\left.\mathcal{I}_{g}\right|^{n+\frac{1}{2}}=\left.\frac{v_{g}(t)}{R_{g}}\right|_{t=(n+1 / 2) \Delta t}
$$

denotes the time-discretized nominal current delivered by the source. The total voltage and current signals excited at the input port of the antenna are then computed as

$$
\begin{aligned}
v_{\text {in }}(t)= & -\int_{C_{V}} \boldsymbol{E}(\boldsymbol{r}, t) \cdot \boldsymbol{d} \boldsymbol{l} \simeq-\left.\mathcal{E}_{z}(t)\right|_{i_{g}, j_{g}, k_{g}+\frac{1}{2}} \\
i_{\text {in }}(t)= & \oint_{C_{I}} \boldsymbol{H}(\boldsymbol{r}, t) \cdot \boldsymbol{d} \boldsymbol{l} \\
\simeq & \left.\mathcal{H}_{y}\right|_{i_{g}+\frac{1}{2}, j_{g}, k_{g}+\frac{1}{2}} ^{n+\frac{1}{2}}-\left.\mathcal{H}_{y}\right|_{i_{g}-\frac{1}{2}, j_{g}, k_{g}+\frac{1}{2}} ^{n+\frac{1}{2}}+ \\
& -\left.\mathcal{H}_{x}\right|_{i_{g}, j_{g}+\frac{1}{2}, k_{g}+\frac{1}{2}} ^{n+\frac{1}{2}}+\left.\mathcal{H}_{x}\right|_{i_{g}, j_{g}-\frac{1}{2}, k_{g}+\frac{1}{2}} ^{n+\frac{1}{2}}
\end{aligned}
$$

where $C_{V}$ is an open contour extending along the driving point, and $C_{I}$ a closed contour path wrapping around the generator. Under the mentioned assumptions, the normalized incident and reflected waves are evaluated as

$$
\begin{aligned}
& a_{i n}(f)=\frac{1}{2}\left[\frac{V_{i n}(f)}{\sqrt{Z_{0}}}+I_{i n}(f) \sqrt{Z_{0}}\right] \\
& b_{i n}(f)=\frac{1}{2}\left[\frac{V_{i n}(f)}{\sqrt{Z_{0}}}-I_{i n}(f) \sqrt{Z_{0}}\right]
\end{aligned}
$$

where $Z_{0}=R_{g}$ denotes the reference impedance and

$$
\begin{aligned}
V_{i n}(f) & =\mathrm{F}\left[v_{i n}(t)\right] \\
I_{i n}(f) & =\mathrm{F}\left[i_{i n}(t)\right]
\end{aligned}
$$

$F[\cdot]$ being the Fourier transform operator. Therefore, the antenna input reflection coefficient can be easily determined as

$$
\Gamma_{i n}(f)=\frac{b_{i n}(f)}{a_{i n}(f)} .
$$

A ten-cell uniaxial perfectly matched layer (UPML) absorbing boundary condition for lossy media [18] has been used at the outer FDTD mesh boundary to simulate the extension of the space lattice to infinity. As outlined in [18], the UPML is indeed perfectly matched to the inhomogeneous medium formed by the upper air region and the lossy material modeling the skin. So, no spurious numerical reflections take place at the air-ground interface. In particular, a quartic polynomial grading of the UPML conductivity profile has been selected in order to have a nominal reflection error $R_{P M L} \simeq e^{-16}$.

By using the presented electromagnetic field prediction model, it has been numerically found that the needle probe having length $l_{p}=0.5 \mathrm{~mm}$ and wire radius $r_{p}=10 \mu \mathrm{m}$ is well-matched to the feeding line in the frequency band around $f_{0} \simeq 139.5 \mathrm{GHz}$ (see Fig. 3). In particular, the central resonant frequency $f_{0}$ of the sensor can be conveniently controlled by 


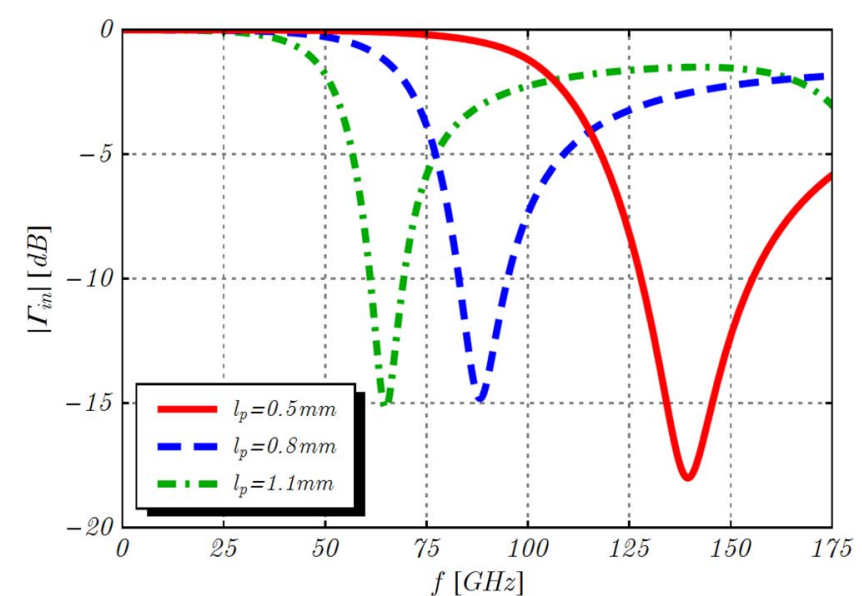

Fig. 3. Frequency-domain behavior of the input reflection coefficient featured by the monopole-like antenna sensor for different lengths of the probe.
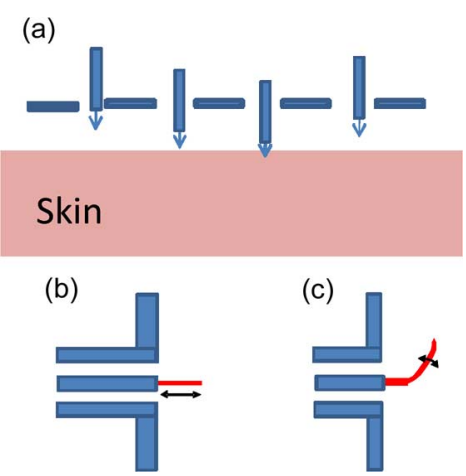

Fig. 4. Reconfigurable sensor for (a) melanoma skin cancer detection with (b) adjustable probe length and (c) curvature.

tuning the length of the radiating flair [see Fig. 4(b)]. As it appears from Fig. 3, an excellent input impedance matching around 88.5 and $65.0 \mathrm{GHz}$ is obtained where the probe length is selected to be $l_{p}=0.8 \mathrm{~mm}$ and $l_{p}=1.1 \mathrm{~mm}$, respectively. In this way one can enhance the radiated field level, and hence increase the accuracy in the scan of anomalous areas on the skin surface. Furthermore, in order to optimize the target detectability over a large area the probe can be bent as shown in Fig. 4(c). A suitable MEMS-based antenna layout for reconfigurable microsensors is described in the following section.

In Fig. 5, the transient and frequency-domain behavior of the copolarized component of the electric field excited along the broadside direction at a distance of $3 \mathrm{~mm}$ from the driving point of the considered antenna sensor is shown for different lengths of the probe. As it can be noticed, the radiated pulse features a reasonably small late-time ringing, which is in turn very important to prevent the masking of the target. A specific parameter study has been finally carried out in order to investigate of the probe response as a function of the relative variation $\Delta \varepsilon_{r} / \varepsilon_{r}$ of the permittivity of the skin due to the presence of the melanoma. In particular, Fig. 6 shows the frequency-domain behavior of the input reflection coefficient of the probe having length $l_{p}=0.5 \mathrm{~mm}$. As it can be noticed in Figs. 6 and 7 , the fundamental resonant frequency of the antenna sensor is pretty stable against relative variations of the permittivity,

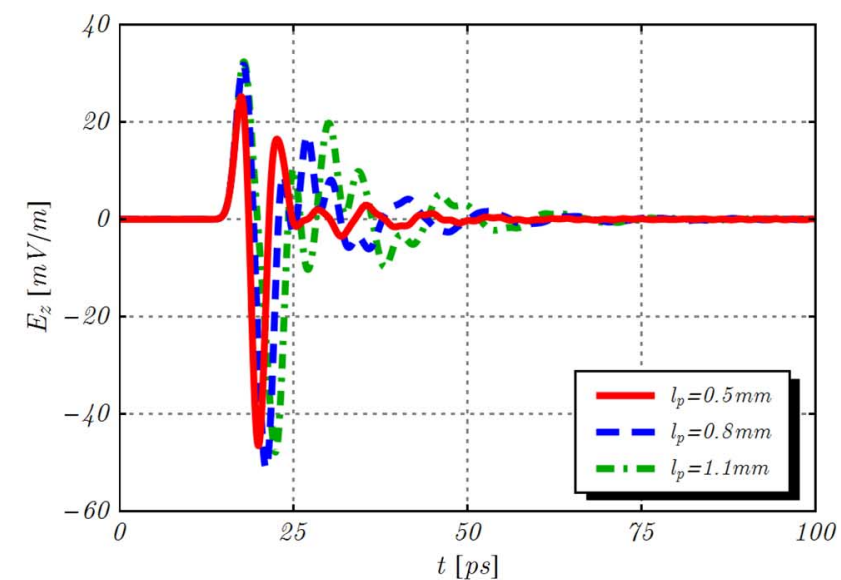

(a)

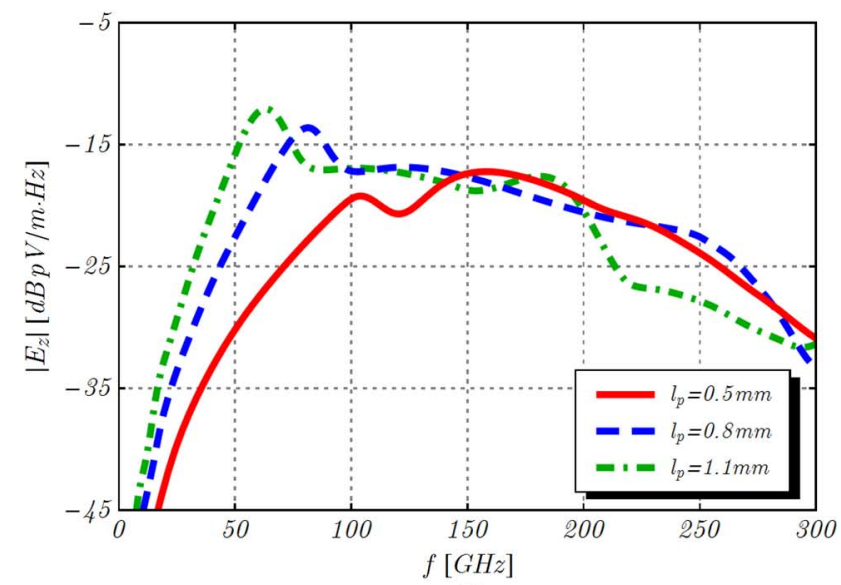

(b)

Fig. 5. (a) Transient and (b) frequency-domain behavior of the copolarized electric field component excited by the antenna sensor at a distance of $3 \mathrm{~mm}$ from the driving point on the surface of the skin.

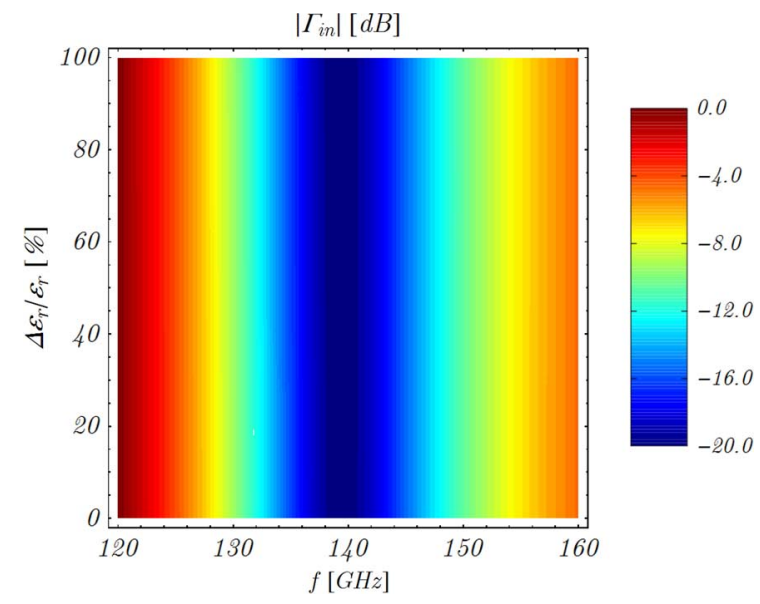

Fig. 6. Frequency-domain behavior of the input reflection coefficient featured by the monopole-like antenna sensor as function of the relative variation $\Delta \varepsilon_{r} / \varepsilon_{r}$ of the permittivity of the skin due to the presence of the melanoma. The probe, having length $l_{p}=0.5 \mathrm{~mm}$, is at distance $d_{s}=1.5 \mathrm{~mm}$ from the surface of the skin (see Fig. 1).

whereas the bandwidth at 10-dB return-loss level slightly increases as $\Delta \varepsilon_{r} / \varepsilon_{r}$ becomes larger. Hence, the detection of possible anomalous areas on the skin surface can be achieved by properly tuning the antenna central working frequency, and by using a suitable high-sensitivity receiver (see the inset of Fig. 7). 


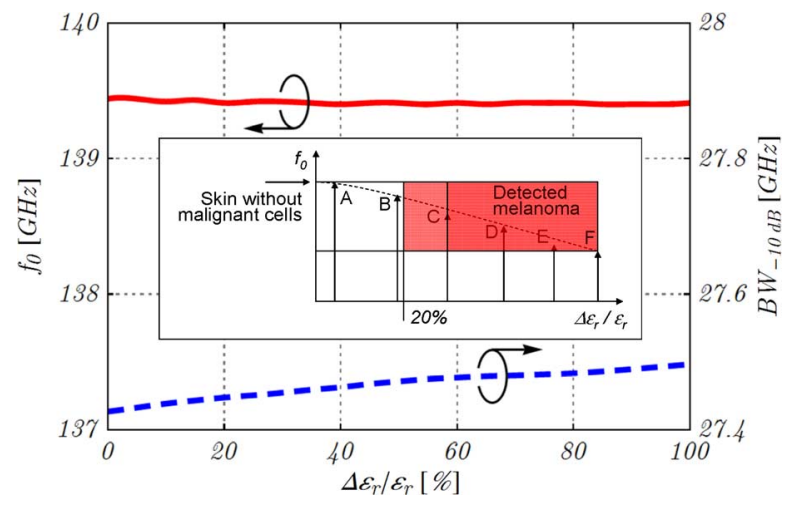

Fig. 7. Fundamental resonant frequency and bandwidth at return-loss level featured by the monopole-like antenna sensor as function of the relative variation $\Delta \varepsilon_{r} / \varepsilon_{r}$ of the permittivity of the skin. The probe, having length $l_{p}=0.5 \mathrm{~mm}$, is at a distance $d_{s}=1.5 \mathrm{~mm}$ from the surface of the skin (see Fig. 1). In particular, the resonant frequency points $A, B, \ldots$ relevant to different risk levels (see inset) can be discriminated by using a suitable high-sensitivity receiver.
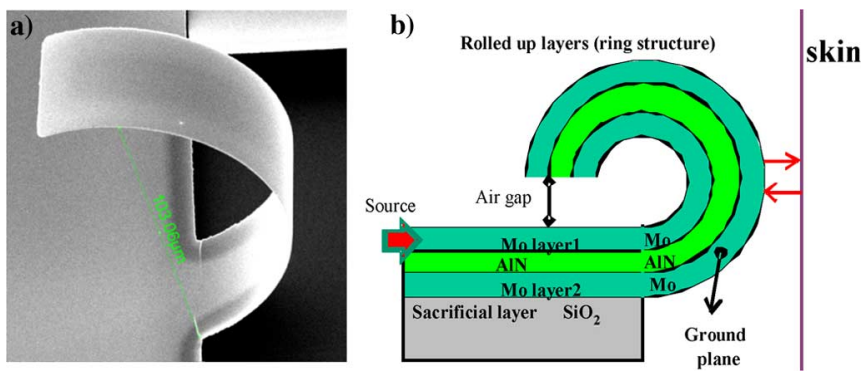

Fig. 8. (a) Scanning electron microscope image and (b) layout of a free-standing MEMS-based microsensor for non-invasive melanoma skin cancer detection. The AIN film, having thickness of $500 \mathrm{~nm}$, is sandwiched between two Molybdenum electrodes.

\section{MEMS-BASED TECHNOLOGICAL IMPLEMENTATION: EXAMPLE OF RECONFIGURABLE ANTENNA SENSOR LAYOUT FOR NEAR-FIELD DETECTION}

In this section, a suitable antenna layout for the proposed reconfigurable microsensors is discussed in details. To this end, we turn to the consideration of the MEMS-based structure shown in Fig. 8. An AlN thin film, sandwiched between two Molybdenum $(\mathrm{Mo})$ electrodes, is grown on a substrate consisting of a thin $\mathrm{SiO}_{2}$ sacrificial layer on a thick Silicon substrate. Molybdenum is chosen as electrode material because of its capability to promote a preferential orientation of the $A l N$ film and its low resistivity. The $A l N$ and $M o$ films are grown by magnetron sputtering. The growth conditions are optimized in order to achieve a good compromise between adhesion stability, crystallographic quality and high residual stress of the films. Probes with different geometries (width of $40-50 \mu \mathrm{m}$ and length ranging from $250 \mu \mathrm{m}$ to $2.5 \mathrm{~mm}$ ) have been manufactured by using conventional micromachining techniques based on the etching of the sacrificial oxide layer. In this way, the free-standing structure turns to consist of an AlN thin layer between two Molybdenum electrodes.

Optical lithography and well-controlled etching procedures are used to define the geometrical characteristics of the microsensor. In particular, a hydrogen fluoride-based solution is used to remove the $\mathrm{SiO}_{2}$ sacrificial layer allowing the release of the strip from the substrate surface. The residual stress of the layered structure results in the rolling-up of the probe (see
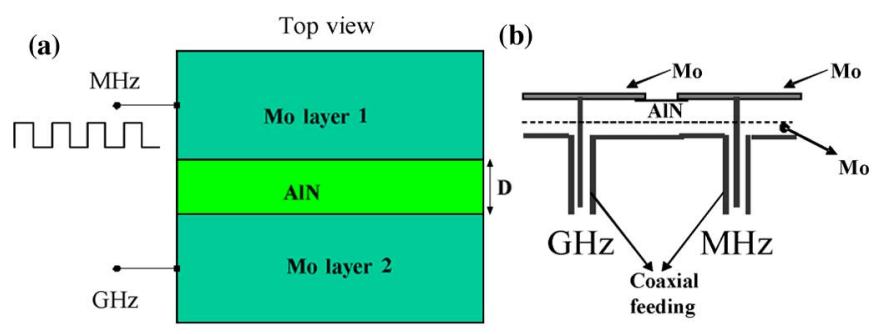

(c)

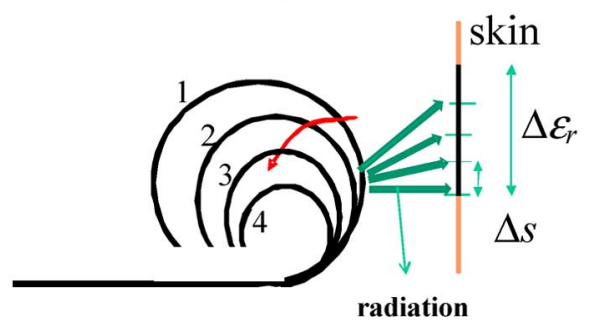

Fig. 9. Possible control mechanism of the first Mo electrode in the (a) proposed MEMS-based microantenna sensor, and (b) relevant coaxial feeding structure: a suitable voltage signal in the $\mathrm{MHz}$ frequency range is used to reconfigure the (c) antenna. In this way, the scanning process of the skin is function of the applied control voltage and the distance between the probe and the skin surface. Technological aspects regarding the coupling of the radio source are under investigation.

Fig. 8). The considered sensor layout can be usefully adopted for a convenient modulation of the transmitted radio signal. In particular, the piezoelectric property of the electrodes forming the structure can be exploited to tune the resonant frequency of the antenna. In fact, as it can be readily inferred, the curvature of the probe arm, controlled by a suitable radio signal, directly affects the antenna radiation impedance and, hence, the phase and/or amplitude of the transmitted electromagnetic field. A possible control mechanism of the Mo electrode in the proposed MEMS-based microantenna sensor is detailed in Fig. 9. In this context, the locally conformal FDTD model considered in this paper can be extended by including the piezoelectric effect through the constitutive relationships between the stress $\mathbf{T}$, the strain $\mathbf{S}$, the electric field $\mathbf{E}$, and the electric displacement D

$$
\left\{\begin{array}{l}
\mathbf{T}=\boldsymbol{c}_{E} \cdot \mathbf{S}-\boldsymbol{e}^{T} \cdot \mathbf{D} \\
\mathbf{D}=\boldsymbol{e} \cdot \mathbf{S}-\varepsilon_{r} \cdot \mathbf{E}
\end{array}\right.
$$

where $c_{E}, e$, and $\varepsilon_{r}$ denote the elasticity, coupling and relative permittivity tensors, respectively, describing the mechanical and electrical properties of the material forming the antenna under analysis. In this way, the modulation mechanisms of the radiated electromagnetic field can be accurately modeled.

It is to be pointed out that the reconfigurability of the probe sensor due to the piezoelectric characteristics of the Mo electrodes allows a versatile scanning of the skin surface. In particular, a maximum probe displacement of 10-20 $\mu \mathrm{m}$ has been experimentally achieved by applying a control voltage of 10-30 V (see Fig. 9). This property can be usefully exploited to detect small variations $\Delta \varepsilon_{r}$ of the permittivity, as well as to carry out an accurate analysis of the spatial extension of anomalies on the surface of the skin. As outlined in Fig. 10, such analysis can be performed by steps $\Delta s$ tuned in such a way as to match the spatial increments of the FDTD grid adopted for the electromagnetic field computations (see Fig. 2). In this way, one can easily monitor the shape and contour of anomalous areas, which 


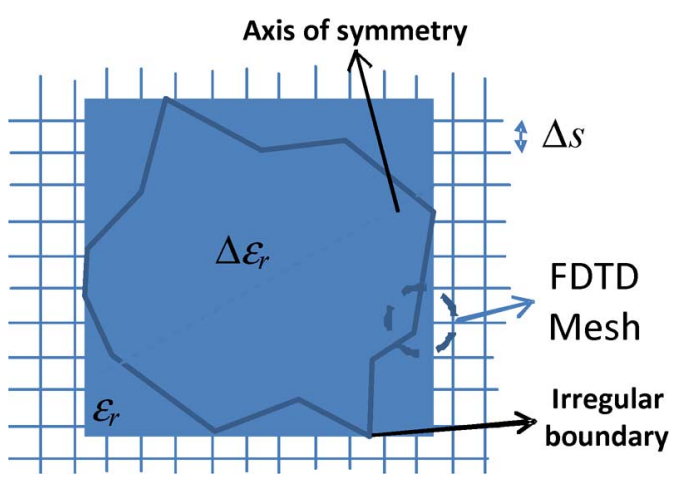

Fig. 10. Grid-based scanning process of melanoma-related anomalies of the skin by means of the proposed reconfigurable microprobe sensor.

are important parameters in the early recognition of malignant melanomas [21].

\section{CONCLUSION}

The full-wave electromagnetic characterization of reconfigurable antenna sensors for the detection of melanoma-related anomalies of the skin has been presented. To this end, an enhanced locally conformal FDTD procedure has been adopted. In this way, a detailed and insightful understanding of the physical processes responsible for the performance of considered class of devices can be achieved. This in turn is very important in order to enhance the structure reliability, optimizing the design cycle.

By using the detailed approach, the accurate time-domain modeling of monopole-like microsensors useful to detect local variations of the electrical properties of the skin tissue due to anomalous cells has been carried out. In this way, one can retrieve information about the growth rate of possible melanomas, which is a very important aspect regarding the monitoring of the risk. It has been numerically found that the resonant frequency of the considered sensors can be conveniently controlled by tuning the length of the antenna flair. This provides an effective means to discriminate working frequency points relevant to different risk levels by using high-sensitivity receivers.

A suitable antenna layout for the proposed reconfigurable detection system has been finally discussed in details. In particular, piezoelectric electrodes controlled by a low-frequency radio signal are used to tune the effective size of the radiating flair and, hence, the fundamental resonant frequency of the sensor. In this context, it is to be pointed out that the presented locally conformal FDTD model can be extended by including the piezoelectric effect through the constitutive relationships describing the mechanical and electrical properties of the materials forming the considered class of MEMS-based probes.

In future studies, an extensive analysis of the probe feeding technique, as well as a detailed investigation of the radio control mechanism of the piezoelectric electrodes in the proposed reconfigurable microsensor layout are needed in order to fully assess the suitability and robustness of the considered devices for non-invasive detection of melanoma-related anomalies of the skin at Terahertz frequencies. Such studies are preliminary to the experimental verification, and assessment of the detection accuracy in practical clinical cases.

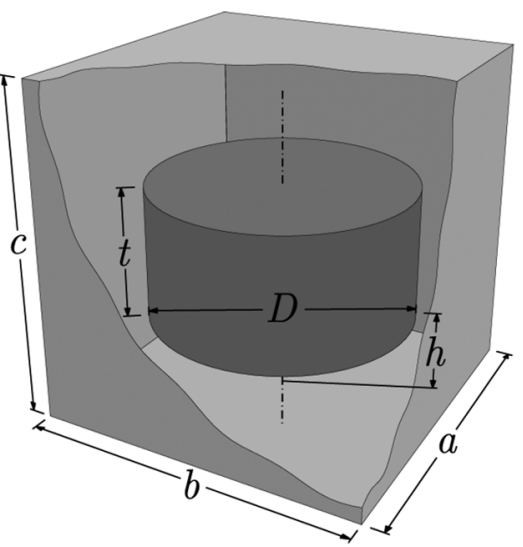

(a)

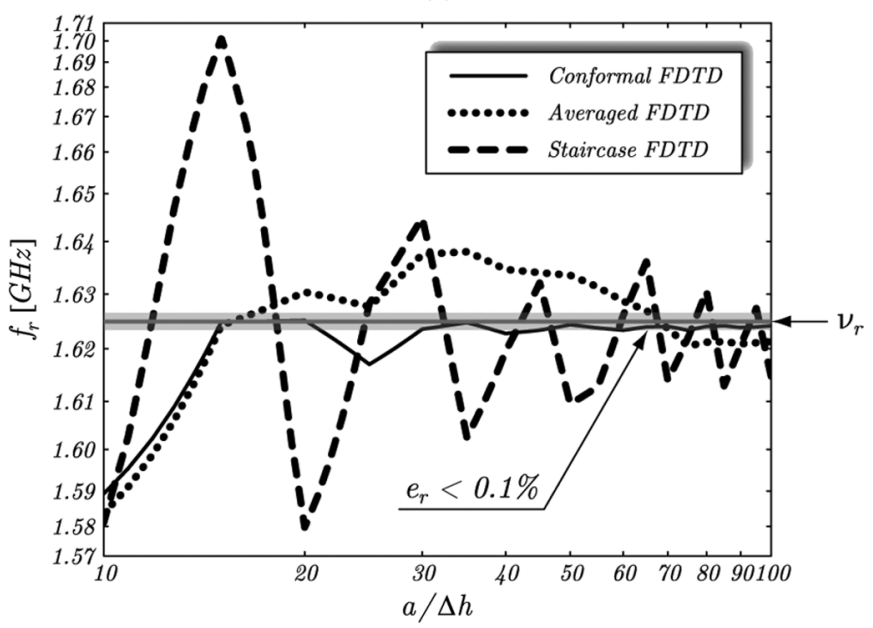

(b)

Fig. 11. (a) Geometry of a dielectric loaded rectangular cavity, and (b) behavior of the relevant fundamental resonant frequency $f_{r}$ as function of the FDTD mesh size $\Delta h$. Shown is the confidence region where the relative error $e_{r}$ with respect to the reference resonant frequency $\nu_{r}=1.625 \mathrm{GHz}$ [22] is smaller than $0.1 \%$. Structure characteristics: $a=b=50 \mathrm{~mm}, c=30 \mathrm{~mm}, D=$ $36 \mathrm{~mm}, t=16 \mathrm{~mm}, h=7 \mathrm{~mm}$. Relative permittivity of the dielectric puck: $e_{r}=37$.

\section{APPENDIX}

In order to validate the accuracy of the proposed locally conformal FDTD scheme, a number of test cases have been considered.

Here the results obtained for the computation of the fundamental resonant frequency of a dielectric resonator enclosed in a metallic cavity are presented. The structure under consideration [see Fig. 11(a)] has been already analyzed in [22]. It consists of a perfectly conducting metallic cavity of dimensions $a=b=50 \mathrm{~mm}$ and $c=30 \mathrm{~mm}$, loaded with a cylindrical dielectric (ceramic) puck having diameter $D=36 \mathrm{~mm}$, height $t=16 \mathrm{~mm}$, and relative dielectric constant $\varepsilon_{r}=37$. The puck is suspended at a distance of $h=7 \mathrm{~mm}$ from the bottom of the cavity. Since the dielectric permittivity of the resonator is rather high, the effect of the orthogonal Cartesian mesh being not conform to the resonator shape is expected to be noticeable. Here, the structure is analyzed by means of a standard FDTD scheme featuring the traditional staircase approximation of the resonator's contour, and by means of the weighted averaging approach proposed in [20], and the locally 
conformal FDTD technique detailed in Section III. The numerical results obtained from these FDTD schemes are compared against the ones reported in [22] resulting from the use of a commercial transmission line matrix (TLM) method-based solver. To this end, a cubic FDTD mesh having fixed spatial increment $\Delta h$ has been adopted to analyze the structure. As it appears in Fig. 11(b), this example clearly demonstrates the suitability of the proposed approach to efficiently handle complex metal-dielectric structures with curved boundaries. The proposed locally FDTD scheme introduces a significant improvement in accuracy over the staircasing approximation, converging very quickly to the reference value. Such feature is thus of crucial importance to optimize the design of complex sensors for real-time diagnosis and monitoring of different types of malignant cancers, such as melanomas with predominant growth underneath the skin surface, whose detection with traditional techniques based on dermatoscopic exams may fail.

\section{REFERENCES}

[1] X.-Q. Li, Q.-X. Liu, X.-J. Wu, L. Zhao, J.-Q. Zhang, and Z.-Q. Zhang, "A GW level high-power radial line helical array antenna," IEEE Trans. Antennas Propagat., vol. 56, no. 9, pp. 2943-2948, Sep. 2008.

[2] R. L. Clark G and R. N. Dean, "Three Dimensional Micromachined Electromagnetic Device and Associated Methods," U.S. patent US 6,271,802 B1, Aug. 7, 2001.

[3] J. M. Bell and M. F. Iskander, "A low-profile archimedean spiral antenna using an EBG ground plane," IEEE. Antennas Wireless Propagat. Letters, vol. 3, no. 1, pp. 223-226, Dec. 2004.

[4] K. Hirose, A. Kimizuka, and H. Nakano, "Microstrip-line antennas with loop elements," in Proc. IEEE Int. Antennas Propagat. Symp., 2007, pp. 721-724.

[5] D. Mittleman and K. Wang, "A Method for Coupling Terahertz Pulses Into a Coaxial Waveguide," Int. patent, WO 2006/019776 A2.

[6] C. W. Jung, M.-J. Lee, G. P. Li, and F. De Flaviis, "Reconfigurable scan-beam single-arm spiral antenna integrated with RF-MEMS switches," IEEE Trans. Antennas Propagat., vol. 54, no. 2, pp. 455-463, Feb. 2006.

[7] A. Massaro, I. Ingrosso, C. Giordano, M. T. Todaro, R. Cingolani, M. De Vittorio, and A. Passaseo, "Micromachining Mo/AlN/Mo piezoelectric ring resonators," in Proc. 39th Eur. Microw. Conf., 2009, pp. $1646-1649$.

[8] W. Xia, K. Saito, M. Takahashi, and K. Ito, "Performance of an implanted cavity slot antenna embedded in the human arm," IEEE Trans. Antennas Propagat., vol. 57, no. 4, pp. 894-899, Apr. 2009.

[9] R. N. Simons, F. A. Miranda, and J. D. Wilson, "Wearable wireless telemetry system for implantable bio-MEMS sensor," in Proc. 28th IEEE EMBS Conf., 2006, pp. 6245-6248.

[10] P. Soontornpipit, C. M. Furse, and Y. C. Chung, "Design of implantable microstrip antenna for communication with medical implants," IEEE Trans. Microw. Theory Tech., vol. 52, no. 8, pp. 1944-1951, Aug. 2004

[11] L. S. Taylor, "Implantable radiators for cancer therapy by microwave hyporthermia," Proc. IEEE, vol. 68, no. 1, pp. 142-149, Jan. 1980.

[12] P. Mehta, K. Chand, D. Narayanswammy, D. G. Beetner, R. Zoughi, and W. V. Stoeker, "Microwave reflectometry as a novel diagnostic tool for detection of skin cancers," IEEE Trans. Instrum. Meas., vol. 55, no. 4, pp. 1309-1316, Aug. 2006.

[13] R. F. Donnelly, M. J. Garland, D. I. J. Morrow, K. Migalska, T. R. R. Singh, R. Majithia, and A. D. Woolfson, "Optical coherence tomography is a valuable tool in the study of the effects of microneedle geometry on skin penetration characteristics and in-skin dissolution," $J$. Control. Release, 2010, 10.1016/j.jconrel.2010.08.008.

[14] A. G. Uribe, J. M. Hong, J. Zou, and L. V. Wang, "Micromachined oblique incidence reflectometry (OIR) probe for skin cancer detection," in Proc. Int. Solid-State Sens., Actuators Microsyst. Conf., 2007, pp. 1099-1102.
[15] V. P. Wallace, A. J. Fitzgerald, E. Pickwell, R. J. Pye, P. F. Taday, N. Flanagan, and T. Ha, "Terahertz pulsed spectroscopy of human basal cell carcinoma," Appl. Spectr., vol. 60, no. 10, pp. 1127-1133, 2006.

[16] B. Freer and J. Venkataraman, "Bio-compatible implantable micro-LC sensor for tissue characterization," in Proc. IEEE AP-S/URSI Symp., 2009, pp. 1-4.

[17] K. S. Yee, "Numerical solution of initial boundary value problems involving Maxwell's equations," IEEE Trans. Antennas Propagat., vol. 14, no. 3, pp. 302-307, May 1966.

[18] A. Taflove and S. C. Hagness, Computational Electrodynamics: The Finite Difference Time Domain Method, 3rd ed. Norwood, MA: Artech House, 2005.

[19] D. Caratelli and R. Cicchetti, "A full-wave analysis of interdigital capacitors for planar integrated circuits," IEEE Trans. Magn., vol. 39, no. 3, pp. 1598-1601, May 2003.

[20] S. Dey and R. Mittra, "A conformal finite-difference time-domain technique for modeling cylindrical dielectric resonators," IEEE Trans. Microw. Theory Tech., vol. 47, no. 9, pp. 1737-1739, Sep. 1999.

[21] W. Stolz, A. Riemann, and A. Cognetta et al., "ABCD rule of dermatoscopy: A new practical method for early recognition of malignant melanoma," Eur. J. Dermatol., vol. 4, pp. 521-527, 1994.

[22] J. Chuma, C. W. Sim, and D. Mirshekar-Syahkal, "Computation of resonant frequencies of dielectric loaded rectangular cavity using TLM method," IET Electron. Lett., vol. 35, no. 20, pp. 1712-1713, Sep. 1999.

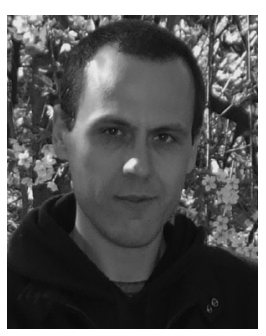

Diego Caratelli was born in Latina, Italy, on May 2, 1975. He received the Laurea (summa cum laude) and Ph.D. degrees in electronic engineering from "La Sapienza" University of Rome, Rome, Italy, in 2000 and 2004, respectively.

In 2005, he joined as a Contract Researcher the Department of Electronic Engineering, "La Sapienza" University of Rome. Since 2007, he has been with the International Research Center for Telecommunications and Radar (IRCTR), Delft University of Technology, Delft, The Netherlands, as a Senior Researcher. His main research activities include the design, analysis, and experimental verification of printed microwave and millimeter-wave passive devices and wideband antennas for satellite, WLAN and GPR applications, the development of analytically based numerical techniques devoted to the modeling of electromagnetic field propagation and diffraction processes, as well as the analysis of EMC/EMI problems in sensitive electronic equipment.

Dr. Caratelli was the recipient of the Young Antenna Engineer Prize at the 32th European Space Agency Antenna Workshop. He received the 2010 Best Paper Award from the Applied Computational Electromagnetics Society (ACES). He serves as reviewer for several international journals, and is a member of ACES and the Italian Electromagnetic Society (SIEm).

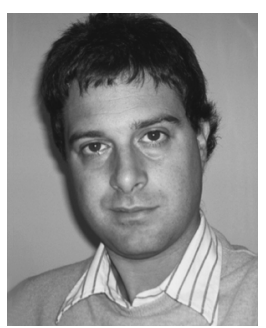

Alessandro Massaro received the Laurea degree in electronic engineering and the Ph.D. degree in telecommunication engineering from the Università Politecnica delle Marche, Ancona, Italy, in 2001 and 2004, respectively

From 2004 to 2006, he worked as a Research Scientist (post-doc) in the Department of Electromagnetism and Bioengineering, Università Politecnica delle Marche. In 2006, he spent two years in research and development in medical and industrial optics industry (endoscope design and optical systems). He worked for two years with the National Nanotechnology Laboratory of CNR-INFM as a Principal Investigator. He is currently a Team Leader in the Robotics Lab. 1, Center of Bio-Molecular Nanotechnology, Italian Institute Technology (IIT), Arnesano, Lecce, Italy. His research interests are in the design and modeling of photonic band gap circuits, in the development of computer-aided design (CAD) tools in the area of integrated optics, MEMS/NEMS technology and systems, and smart material design. Actually, he is a member of the European Microwave Association (EuMA). 


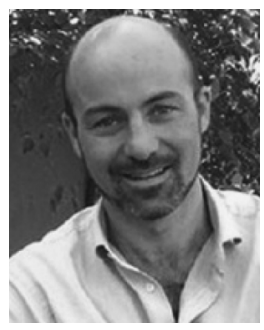

Roberto Cingolani was born in Milan, Italy, in December 1961. He received the Ph.D. degree in physics from Scuola Normale Superiore di Pisa, Pisa, Italy.

He was a staff member of the Max Planck Institute $F K F$-Stuttgart from 1989 to 1991 . He was a Visiting Scientists at Tokyo University, Tokyo, Japan, in 1997, and appointed a Full Professor of experimental physics at the University of Salento in 2000. He has been a Scientific Director of the Italian Institute of Technology since 2004. He is an author or coauthor of more than 500 papers in international journals and he holds 20 patents. His research activity includes nanotechnology and nanofabrication, semiconductor physics and devices, molecular organic nanostructures and devices, and bio-nanotechnologies.

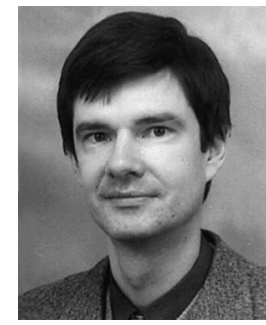

Alexander G. Yarovoy (M'96-SM'04) received the Diploma degree (with honors) in radiophysics and electronics from Kharkov State University, Kharkov, Ukraine, in 1984 and the Candidate Phys. and Math. Sci. (Ph.D. equivalent) and Doctor Phys. and Math. Sci. (D.Sc. equivalent) degrees in radiophysics in 1987 and 1994, respectively.

In 1987, he joined the Department of Radiophysics, Kharkov State University, as a Researcher and became a Professor there in 1997. From September 1994 to 1996, he was with the Technical University of Ilmenau, Ilmenau, Germany, as a Visiting Researcher. Since 1999, he has been with the International Research Centre for Telecommunications and Radar (IRCTR), Delft University of Technology, Delft, the Netherlands. Since 2009, he leads there a chair of Microwave Technology and Systems for Radar. His main research interests are in ultrawideband (UWB) microwave technology and its applications (in particular, UWB radars) and applied electromagnetics (in particular, UWB antennas).

Prof. Yarovoy was the recipient of a 1996 International Union of Radio Science (URSI) "Young Scientists Award" and the European Microwave Week Radar Award in 2001 for the paper that best advances the state-of-the-art in radar technology (together with L. P. Ligthart and P. van Genderen). He served as the Chair of the Fifth European Radar Conference (EuRAD'08), Amsterdam, The Netherlands, and Co-Chairman and the Technical Program Committee Chair of the Tenth International Conference on Ground Penetrating Radar (GPR2004), Delft. 